\title{
Functional marker development of miR1511-InDel and allelic diversity within the genus Glycine
}

\author{
Nang Myint Phyu Sin Htwe ${ }^{1 \dagger}$, Zhong-Qin Luo ${ }^{1 \dagger}$, Long-Guo Jin', Brian Nadon², Ke-Jing Wang ${ }^{1}$ and Li-Juan Qiu ${ }^{1 *}$
}

\begin{abstract}
Background: Single-stranded non-protein coding small RNAs, 18-25 nucleotides in length, are ubiquitous throughout plants genomes and are involved in post-transcriptional gene regulation. Several types of DNA markers have been reported for the detection of genetic diversity or sequence variation in soybean, one of the most important legume crops in worldwide for seed protein and oil content. Recently, with the available of public genomic databases, there has been a shift from the labor-intensive development of PCR-based markers to sequence-based genotyping and the development of functional markers within genes, often coupled with the use of RNA information. But thus far miRNA-based markers have been only developed in rice and tobacco. Here we report the first functional molecular miRNA marker, miR1511-InDel, in soybean for a specific single copy locus used to assess genetic variation in domesticated soybean (Glycine max [L.] Merr) and its wild progenitor (Glycine soja Sieb. \& Zucc.).

Results: We genotyped a total of 1,669 accessions of domesticated soybean (G. max) and its wild progenitor G. soja which are native throughout the China and parts of Korea, Japan and Russia. The results indicate that the miR1511 locus is distributed in cultivated soybean and has three alleles in annual wild soybean. Based on this result, we proposed that miR-InDel marker technology can be used to assess genetic variation. The inclusion of geo-reference data with miR1511-InDel marker data corroborated that accessions from the Yellow River basin (Huanghuai) exhibited high genetic diversity which provides more molecular evidence for gene diversity in annual wild soybean and domestication of soybean.

Conclusions: These results provide evidence for the use of RNA marker, miRNA1511-InDel, as a soybean-specific functional maker for the study of genetic diversity, genotyping of germplasm and evolution studies. This is also the first report of functional marker developed from soybean miRNA located within the functional region of pre-miRNA1511.
\end{abstract}

Keywords: Soybean, miRNA, Genetic diversity, Genetic marker

\section{Background}

Soybean (G. $\max$ ) is one of the most economically important global crops, providing not only proteins and vegetable oils for human beings but also feed for livestock and biofuel feedstock [1]. Moreover, it is one of the most commonly cultivated crops worldwide and is known for its capacity to fix atmospheric nitrogen through symbioses with soil-borne microorganisms [2] and has nutraceutical properties such as isoflavones,

\footnotetext{
*Correspondence: qiulijuan@caas.cn

${ }^{\dagger}$ Equal contributors

${ }^{1}$ The National Key Facility for Crop Gene Resources and Genetic Improvement (NFCRI), Institute of Crop Science, Chinese Academy of Agricultural Sciences, 100081 Beijing, China

Full list of author information is available at the end of the article
}

saponins and tocopherols [3]. Molecular markers are useful tools for basic research and plant breeding to determine the order of genes along the chromosome, detect genetic variability and diversity, construct genetic maps, and track individuals or lines carrying particular genes/loci [4]. In addition, they has been used for studies in molecular ecology, developmental biology, conservation biology, phylogenetics and systematic, as well as in diagnostic of forensic, paternity and diseases assessment [5].

The genetic linkage map in humans using restriction fragment length polymorphism (RFLP) was the one of the first molecular marker applications used in the detection of DNA polymorphism [6]. With the innovation of PCR technology, several alternative DNA

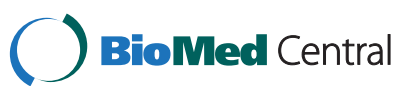


marker techniques such as random amplified polymorphic DNA (RAPD) [7], amplified fragment length polymorphisms AFLP [8], simple sequence repeats (SSR) [9], and single-nucleotide polymorphisms (SNP) [10] were developed. In recent years, technological advances have contributed to advancements in every aspect of molecular marker techniques making them technically initiating a trend away from random DNA markers to functional markers due to rapid growth in genomic research [11]. In soybean, various types of DNA-based markers have been used for linkage mapping [12-14], analysis of genetic variability and diversity $[15,16]$, and identify lines carrying specific genes [17]. Recently, with the availability of genome sequencing in soybean, it has become relatively easy to find single-nucleotide polymorphisms (SNPs) and small insertions/deletions (InDels) which are attractive mapping tools as compared to many other markers types. For example, de novo assembly a pan-genome for wild soybean indicated that three InDels in annual wild soybean (G. soja) may be responsible for changing the growth habit from the twining in G. soja to the erect type in G. $\max$ [18]. Moreover, from the same data set, 0.50 to 0.77 million InDels were detected in G. soja as compared to G. $\max$ [18]. In addition, the usefulness of InDel markers are supported by their wide distribution across the genome and that they are easily assayed by PCR using flanking sequences [19].

MicroRNAs (miRNAs) are a new type of abundant small RNAs 18-25 nucleotides in length [20], and play key roles in plant development $[21,22]$, response to nutrient deficiency [23] and other abiotic stresses [24, 25]. MicroRNAs can be phylogenetically conserved and silence gene expression at the post-transcriptional level by either cleaving or inhibiting translation of a target mRNA via complementation between the miRNA and target mRNA [20, 26, 27]. Recent developments of next generation or deep sequencing technologies have resulted in an increase in microRNA discovery, miRNA detection and analysis [28]. The miRNA Registry Database as of release 21, June 2014 contained 28,645 stem loop miRNAs with 35,825 mature miRNA sequences from 223 species of which there were 573 stem loop miRNAs with 639 mature miRNA sequences for cultivated soybean and 13 stem loop miRNAs with 13 mature miRNA in wild soybean (http://www.mirbase.org/). With the increase in miRNA sequences, it has become possible to develop miRNA-based markers, which is a new type of marker with easy and efficient detection. Using multi-mapped small RNA sequences, inter small RNA polymorphism (iSNP) marker technology was developed for mapping and fingerprinting in species with extremely low genetic diversity [29]. In addition, small RNA-based SSR markers were developed for genetic diversity analysis between salt tolerant and sensitive rice genotypes [30].

miR1511 is a novel small RNA first identified in soybean roots by deep sequencing [31] followed by discovery during stress response in different organs and growth conditions in Phaseolus vulgaris [32], in root and nodules of Medicago truncatula [33], and in various tissue of Vitis vinifera [34]. Base on bioinformatics information, the precursor of pre- miR1511, a $129 \mathrm{bp}$ sequence, is located in an intergenic region on chromosome 18, 10,405 bp downstream of Glyma18g19410.1 and 12,789 bp upstream of Glyma18g19420.1 in William 82. In our previous study, we confirmed the existence of miR1511 and identified its target gene [35]. In this study, we developed a miR1511-InDel marker, which was used to investigate genetic diversity in a large sample of G. max and G. soja. These results provide a reference for miRNA1511-InDel as a new functional marker type for the study of genetic diversity, gene mapping and molecular breeding.

\section{Results}

Functional marker for miR1511/miR1511* allelic variation

To determine allelic variation of soybean subgenus $G$. glycine and G. soja, we developed an Insertion/Deletion marker for miR1511/miR1511* (miR1511-InDel) in the flanking region of stem loop miR1511. We PCR amplified sequences that contained the pre-miR1511 locus and found three fragments sizes ranging from 400 to $600 \mathrm{bp}$. The result showed there were no differences in the size of amplified products in cultivated and perennial wild soybean (Fig. 1a, c), while there were three allelic variants in annual wild soybean (Fig. 1b). The amplified products from the cultivated, annual and perennial wild soybean accessions were verified by DNA sequencing. Sequencing results revealed that the soybean miR1511 mature sequence and miR1511* were found in G. $\max$ and perennial wild soybean (G. microplylla, G. tabacine, G. latifolia, and G. tomentella). There were three types of allelic variants located within the stem loop of miRNA in annual wild soybean (named miR1511-InDel1a, miR1511-InDel-1b, and miR1511-InDel-1c) (Table 1). MiR1511-InDel-1a had a 147 bp deletion including the complete mature sequence of miR1511. MiR1511-InDel$1 b$ had a $71 \mathrm{bp}$ deletion that included parts of both the mature miR1511 and the miR1511* sequence. MiR1511InDel-1c included both mature miR1511 and miR1511* sequences and was identical to cultivated soybean (Fig. 1d). In order to know the feasibility and common of this type of miRNA-InDel marker, 573 stem loop miRNAs with 639 mature miRNA sequences were downloaded from miRBase (http://www.mirbase.org/) and multi aligned with seven G. soja accessions (A to G) in pan-genome [18]. There were more than $10 \%$ (107/ 


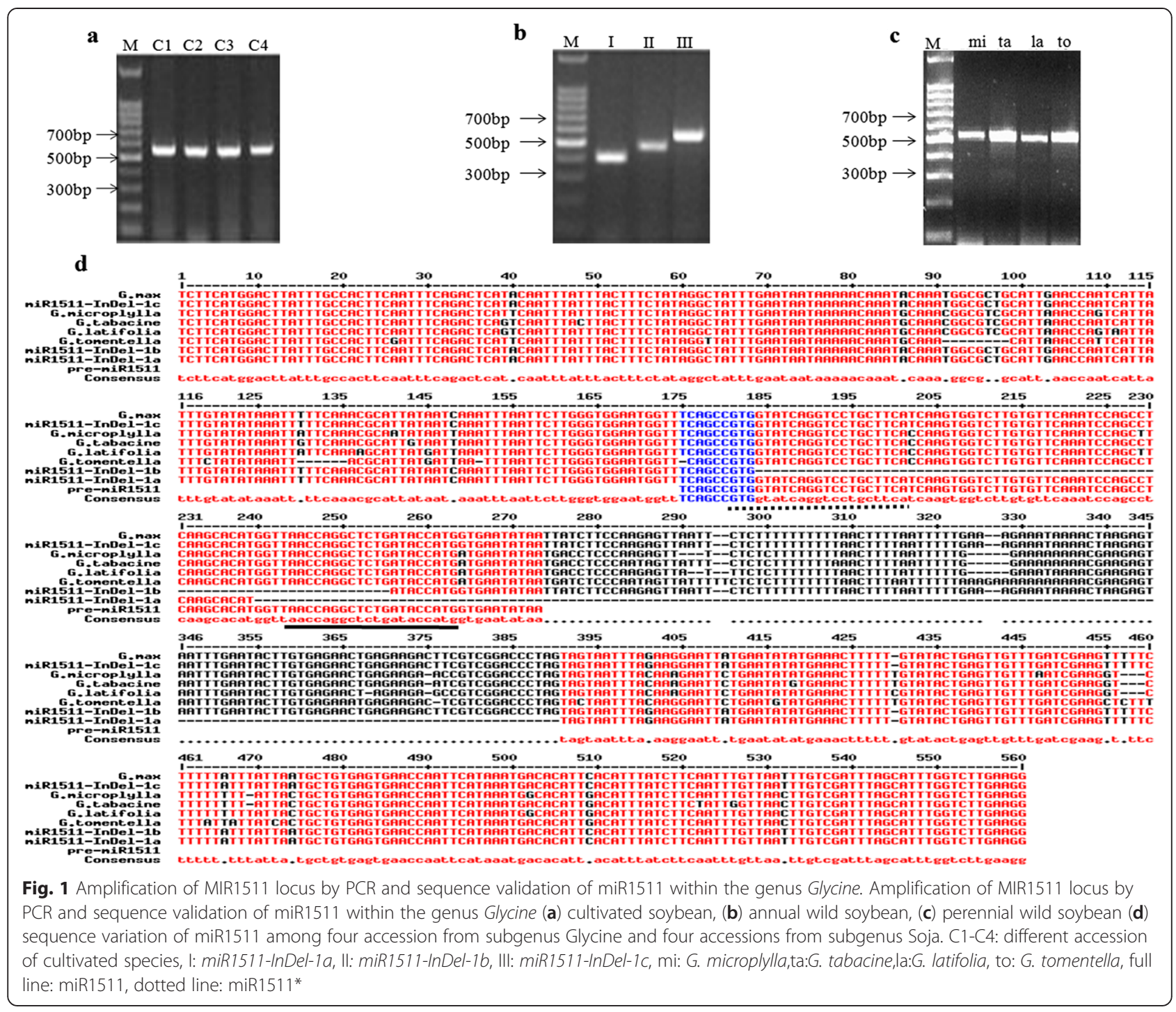

639 mature miRNA) of this type of miRNA-InDel markers. Interestingly, miR4387e and miR4399 have been found to have similar variation as miR1511-InDel that deletion in Yellow river region.

\section{Phylogenetic analysis of miR1511}

In this study, we analyzed including an average $250 \mathrm{bp}$ of $5^{\prime}$ and $3^{\prime}$ flanking sequences of pre- miR1511 in other crops using a low stringency BlastN search with the precursor sequence of soybean miR1511 as a query. The resulting output sequences were inspected for the presence of the mature sequence and then their secondary structures were predicted using the RNAfold program (http://mfold.rna.albany.edu/?q=mfold) (Additional file 1: Figure S1). Except for G. $\max$, the mature sequence of miR1511 is not in the stem region in any legume species including $M$. truncatula, $L$. japonica and two out group species, $P$. trichocarpa and $V$. vinifera (Additional file 2: Figure S2). An un-rooted tree was constructed using the mature sequences of miR1511 among the subgenus Glycine, and two as outgroup which were not in stem region using the maximum likelihood

Table 1 Types of allelic variation in G. Soja

\begin{tabular}{lll}
\hline No. & Name of allele & Difference \\
\hline 1 & miR1511-Indel-1a & Deletion of $147 \mathrm{bp}$ including complete miR1511 mature sequence \\
2 & miR1511-Indel-1b & Deletion of $71 \mathrm{bp}$ including partial of miR1511* (18 bp deletion) and miR1511 (12 bp deletion) mature sequence \\
3 & miR1511-Indel-1c & No InDel and have complete miR1511* (21 bp) and miR1511 (21 bp) mature sequence \\
\hline
\end{tabular}


(ML) method. The observed tree suggested that subgenus Glycine (G. microplylla, G. tabacine, G. latifolia and G. tomentella) and Soja, including cultivated soybean and annual wild soybean, group into one clade with a bootstrap value of $93 \%$ with the exception of miR1511-InDel-1a. G. soja was divided into two groups based on presence or complete deletion of miR1511 sequence. G. soja with either miR1511-InDel-1b or miR1511-InDel-1c were grouped with G. $\max$ with a bootstrap value of $98 \%$ and G. soja with miR1511-InDel-1a was outside the clade (Fig. 2).

\section{Allelic diversity of InDel marker miR1511}

In order to validate the conserved mature miR1511 and miR1511* within G. $\max$, and variation in G. soja, we used a total of 1,206 accessions of cultivated soybean ( $G$. max) including a minicore collection representing four major soybean growing areas of China and 463 accessions of annual wild soybean (G. soja) from three regions of China, Japan, Korea and Russia. All cultivated soybean accessions contained mature miR1511 and miR1511* sequences (data not shown). As mentioned previously, there were three types of alleles in G. soja. MiR1511InDel-1c was the most abundant and distributed throughout China, Japan, Korea and Russia, just as in the cultivated soybean accessions (Fig. 3). However, miR1511-InDel-1a containing a complete deletion of mature miR1511 was found mostly in accessions collected along the Yellow River basin including Gansu (GS), Hebei (HE), Henan (HA), Inner Mongolia (NM), Shandong (SD), and Shanxi (SX); whereas, miR1511InDel-1b, with a partial deletion of both the mature miR1511 and miR1511* sequence, was found in Hunan

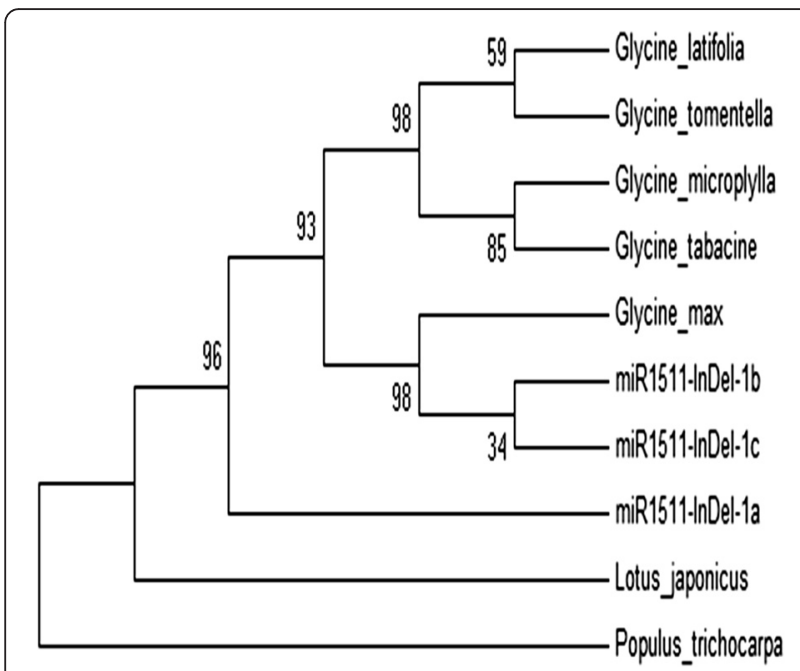

Fig. 2 Phylogenetic analysis of miR1511. Tree constructed from a complete deletion alignment using MEGA 4.0 and the maximum likelihood method with 1,000 bootstrap replicates. The numbers at the nodes represent bootstrap support for the given relationships
(HN) and Hubei (HB) provinces (Fig. 3). Although many accessions were analyzed from the Northeast region of China, e.g. Heilongjiang (HL) and Jilin (JL) provinces, there was only one allele, miR1511-InDel-1c. Incidentally, accession G. soja D from Yellow River basin (Huanghuai), one of seven G. soja accession sequenced as part of a pan-genome, contained the miR1511-InDel-1a allele [18] (Additional file 3: Figure S3). The diversity index was highest in Yellow River basin (Huanghuai) followed by South region of China and Northeast region of China (Table 2). There was no genetic diversity for this locus in accessions of Korea, Japan and Russia. These results provide Yellow River basins (Huanghuai) is the original center for miR1511 locus diversity based on molecular evidence of allelic diversity of miR1511-Indel.

\section{Northern blot and qRT-PCR analysis of miRNAs}

We experimentally verified the presence and absence of miR1511 by Northern blotting using specific probes and U6 snRNA as an internal reference. We randomly selected three accessions each which representing absence and presence of miR1511 in G. soja and Zhongpin 95-5383 (G. max) as control (C) (Fig. 4). In accessions without the deletion of miR1511 and in the control ( $G$. $\max$ ), we detected the signal at $21 \mathrm{bp}$, but no signals were detected in accessions with complete deletions of miR1511 indicating there is no expression in miR1511deleted accessions as compared to G. $\max$ and accessions with no deletions.

In addition, we further provided the present or absent of miNA1511 and miR1511* by qRT-PCR under drought stress in $0,1,3 \mathrm{~h}$ time points respectively. Both miR1511 and miR1511* expressed only in Type III (miR1511-InDel-1c) accession under both control and drought stress condition, and no transcription for type I (miR1511-InDel-1ab) and type II (miR1511InDel-1b) accessions in all conditions (Fig. 5a, b). The melting curve analysis showed two separate peaks for Type I (miR1511-InDel-1a) and Type II (miR1511InDel-1b) accessions at real-time PCR dissociation curve that was completely irreproducible for both miR1511 and miR1511* transcriptions (Fig. 5c). However, there was only one peak for miR1520d which was used as internal control in type I (miR1511-InDel-1a) and Type II (miR1511-InDel-1b) accessions (Fig. 5d). The most possible cause presenting two separate peaks at dissociation curve is non-specific amplification which clearly indicated that no function in type I (miR1511-InDel-1a) and type II (miR1511-InDel-1b) accessions which lack both of miR1511 and miR1511*. This data is also consistent with northern blot confirmation which only showed the signal at $21 \mathrm{bp}$ in type III (miR1511-InDel-1c) accessions (Fig. 4). 


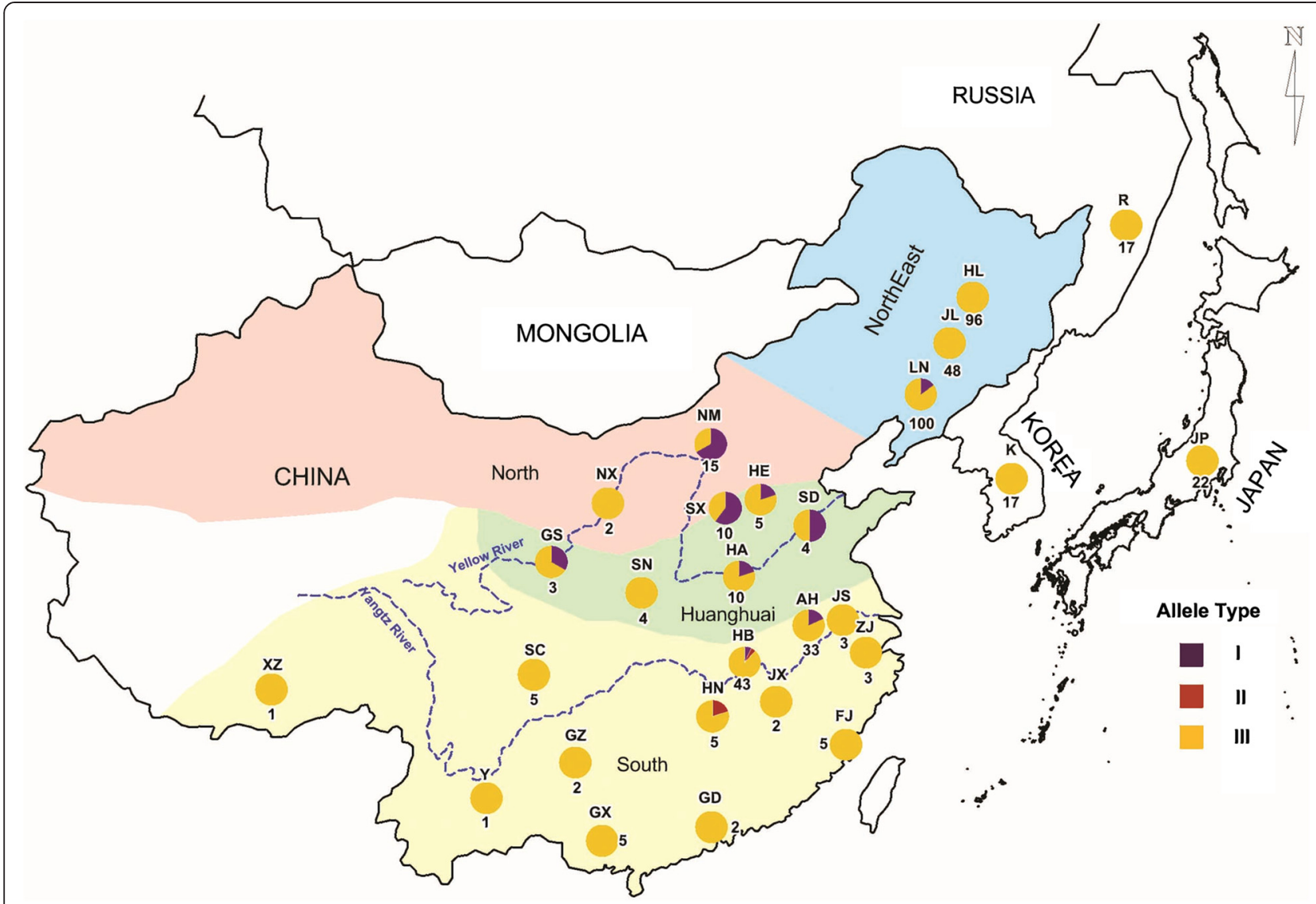

Fig. 3 Geographic distributions of samples (G.soja) used in this study. Each circle represents a different province in China and the country out of China. The percentage of each allele of G. soja is designated by pie chart on the map. The total number of samples per province is indicated by the number. I: miR1511-InDel-1a, II: miR1511-InDel-1b, III: miR1511-InDel-1C, HL: Heilongjiang, JL: Jilin, LN: Liaoning, NM: Neimengu, NX: Ningxia, HE: Hebei, SX: Shanxi, SD: Shangdong, HA: Henan, SN: Shannxi, GS: Gansu, JS: Jiangsu, AH: Anhui, ZJ: Zhejiang, HB: Hubei, JX: Jiangxi, HN: Hunan, FJ: Fujian, GZ: Guizhou, SC: Sichuan, GX: Guangxi, Y: Yunnan, XZ: Xizang, GD: Guangdong

\section{Discussion}

MicroRNA functional markers-miR1511 as an exemplar Mapping markers have evolved from morphological markers to isoenzyme marker to random DNA markers and now to gene targeted functional markers developed from polymorphic sites within genes. Although the use of some recently developed marker techniques in plant sciences is not yet as extensive as that of well established methods such as SSR markers, the number of studies utilizing these advanced methods is rapidly increasing [5]. In this report, we demonstrate four interesting features of miR1511-InDel marker. Firstly, miR1511-InDel is part of a new class of small RNAs (miRNAs) that are single copy number and from which we were able to make inferences about genetic diversity within the subgenus Glycine. Secondly, marker developed from miRNA can be developed easily with efficient detection though detection of miRNA is difficult. It is evidence that this miR1511-InDel marker could be distinguished on the presence or absence of miR1511 due to allelic variation within the pre-miR1511 in soybean germplasm. Thirdly, miR1511-InDel is a functional marker as miRNAs play in functional roles in the regulation of gene expression at the post-transcriptional level in eukaryotes and viruses $[26,36]$. In addition, it has been confirmed that there are anti co-expression between miR1511 and GmRPL4a in different tissue [35]. In addition, there are anti co-expression between miR1511/miR1511* and GmRPL4a under drought treatment (Additional file 4: Figure S4). Recently, it has been reported that loss-of-function mutants of $R P L 4 A$, rpl4a, which were initially identified as a mutant with altered trafficking of vacuolar targeted proteins, display auxin-related developmental defect phenotypes such as narrow pointed first leaves and retarded growth [37]. Fourthly, miR1511-InDel is soybean-specific as confirmed by the existence of complementary strand of miR1511 (miR1511*) and verified by its target cleavage site (GmRPL4a) [35] yet found in any other crop. GmRPL4a belongs to $60 \mathrm{~S}$ ribosomal 
Table 2 Analyses of genetic variance based on miR1511-InDel marker on different classification/Region of soybean

\begin{tabular}{|c|c|c|c|c|c|}
\hline \multirow{2}{*}{$\begin{array}{l}\text { Classification/ } \\
\text { Region }\end{array}$} & \multirow{2}{*}{$\begin{array}{l}\text { No. of } \\
\text { Accessions }\end{array}$} & \multicolumn{3}{|c|}{$\%$ of different type allele } & \multirow{2}{*}{$\begin{array}{l}\text { Diversity } \\
\text { index }\end{array}$} \\
\hline & & $\begin{array}{l}\text { miR1511- } \\
\text { InDel-1a }\end{array}$ & $\begin{array}{l}\text { miR1511- } \\
\text { InDel-1b }\end{array}$ & $\begin{array}{l}\text { miR1511- } \\
\text { InDel-1c }\end{array}$ & \\
\hline $\begin{array}{l}\text { Subgenus } \\
\text { Glycine }\end{array}$ & 4 & 0.00 & 0.00 & 100.00 & 0.00 \\
\hline \multicolumn{6}{|l|}{$\begin{array}{l}\text { Subgenus } \\
\text { Soja }\end{array}$} \\
\hline$\overline{G . \max }$ & 1206 & 0.00 & 0.00 & 100.00 & 0.00 \\
\hline G.soja & 463 & 9.94 & 0.65 & 89.42 & 0.36 \\
\hline \multicolumn{6}{|l|}{ Region } \\
\hline \multicolumn{6}{|l|}{ China } \\
\hline $\begin{array}{l}\text { North east } \\
\text { China }\end{array}$ & 244 & 6.15 & 0.00 & 93.85 & 0.23 \\
\hline $\begin{array}{l}\text { Yellow river } \\
\text { basin }\end{array}$ & 53 & 41.51 & 0.00 & 58.49 & 0.68 \\
\hline South China & 110 & 8.18 & 2.73 & 89.09 & 0.41 \\
\hline Korea & 17 & 0.00 & 0.00 & 100.00 & 0.00 \\
\hline Japan & 22 & 0.00 & 0.00 & 100.00 & 0.00 \\
\hline Russia & 17 & 0.00 & 0.00 & 100.00 & 0.00 \\
\hline
\end{tabular}

protein L4 family and is homologous to RPL4a in Arabidopsis which is involved in plastid transcriptional regulation [38]. Furthermore, there was no sequence match for pre-miR1511 in P. vulgaris (Additional file 3: Figure S3) and the flanking region of target gene cleavage site in $P$. vulgaris was diverged from that of soybean. Moreover, there was no sequence match to mature miR1511 in P.valgaris and there were three SNPs at miR1511* (Additional file 5: Figure S5).

\section{Mode of miRNA evolution or producing}

The first miRNAs, Lin-4 and Let-7, were discovered as the components of developmental pathways in Caenorhabditis elegans [39-41]. Since then many studies have found and elucidated the roles of miRNAs in diverse species, including plants. The evolutionary history of miRNA genes has been well characterized in animals, for example the formation of the miR-17 cluster

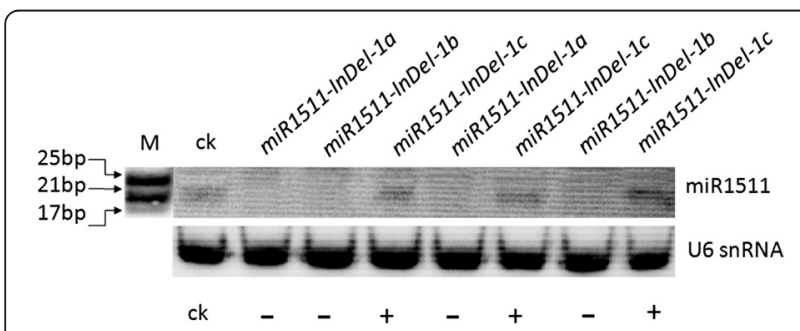

Fig. 4 Small RNA northern blotting analysis of miR1511. Expression of miR1511 in various accessions of G. soja, M: marker; CK: control; deletion of miR1511 (miR1511-InDel-1a); partial deletion of miR1511 (miR1511-InDel-1a); present of miR1511 (miR1511-InDel-1c)
[42] and the imprinted miR-134 gene cluster at human locus 14q32 [43-45]. With the increasing number of identified miRNAs by bioinformatics prediction and deep sequencing, species specific or non conserved miRNAs have been found in rice [46], Medicago truncatula [47] and soybean [31]. It has been suggested that as the majority of these are restricted to closely related species and many could be species specific. Therefore, it is reasonable to hypothesize that plants harbor relatively large numbers of recently born miRNA loci [48]. In plants, some miRNAs and their target genes have been conserved since the last common ancestor of bryophytes and seed plants more than 400 million years ago [49]. Evolution of miRNA genes in plant are thought to originate via three modes: i) produced by inverted duplication of target gene sequences [50]; ii) generated from inverted repeat transposable elements [51]; and iii) spawned from random foldback sequences ("spontaneous evolution") [52]. In this study, the miR1511 foldback lack extended similarity or complementary to the target gene, GmRPL4a, [35] or any other of the predicted target gene sequence. Therefore, it is inconsistent with the hypothesis of inverted duplication in which the foldback arms of recently evolved miRNA genes containing relatively long sequences similar to target gene sequences [50]. Transposable element-derived miRNAs have the potential to regulate multiple genes via homologous target sites dispersed throughout the genome [51]. The target gene of miR1511 has another copy, GmRPL4d, that was not cleaved by miR1511, as determined by $5^{\prime}$ RACE [35], and thus may not be derived from a transposon. It is possible that miR1511 could be derived from random foldback sequences. Thus, by chance, miR1511 could be a newly spawned and species-specific miRNA as even some of the closely related wild species, miR1511InDel-1a, lack the mature miR1511 sequence (Fig. 1).

\section{Diversity or natural selection of miR1511}

It has been established that cultivated soybean (G. $\max$ ) was domesticated from its annual wild relative soybean (G. soja) in East Asia more than 3,000 years ago [53]. SSR, SNP and resequencing indicated that G. soja has greater genetic diversity and higher allelic diversity than G. $\max [16,54]$. Similarly, in this study we found that marker miR1511-InDel can differentiate between the subgenus of Glycine and Soja in which allelic diversity was found only in G. soja (Table. 1). On the basis of the geographical distribution of accessions, the Yellow River basin (Huanghuai) is the most likely centre of diversity where allelic variation, especially as the complete deletion of mature miR1511 in miR1511InDel-1a, was observed mostly in that region (Fig. 3). Moreover, our data is consistent with pan genome analysis of seven wild soybean accessions (G. soja A to G) accessions [18] in which only G. soja D from Yellow 


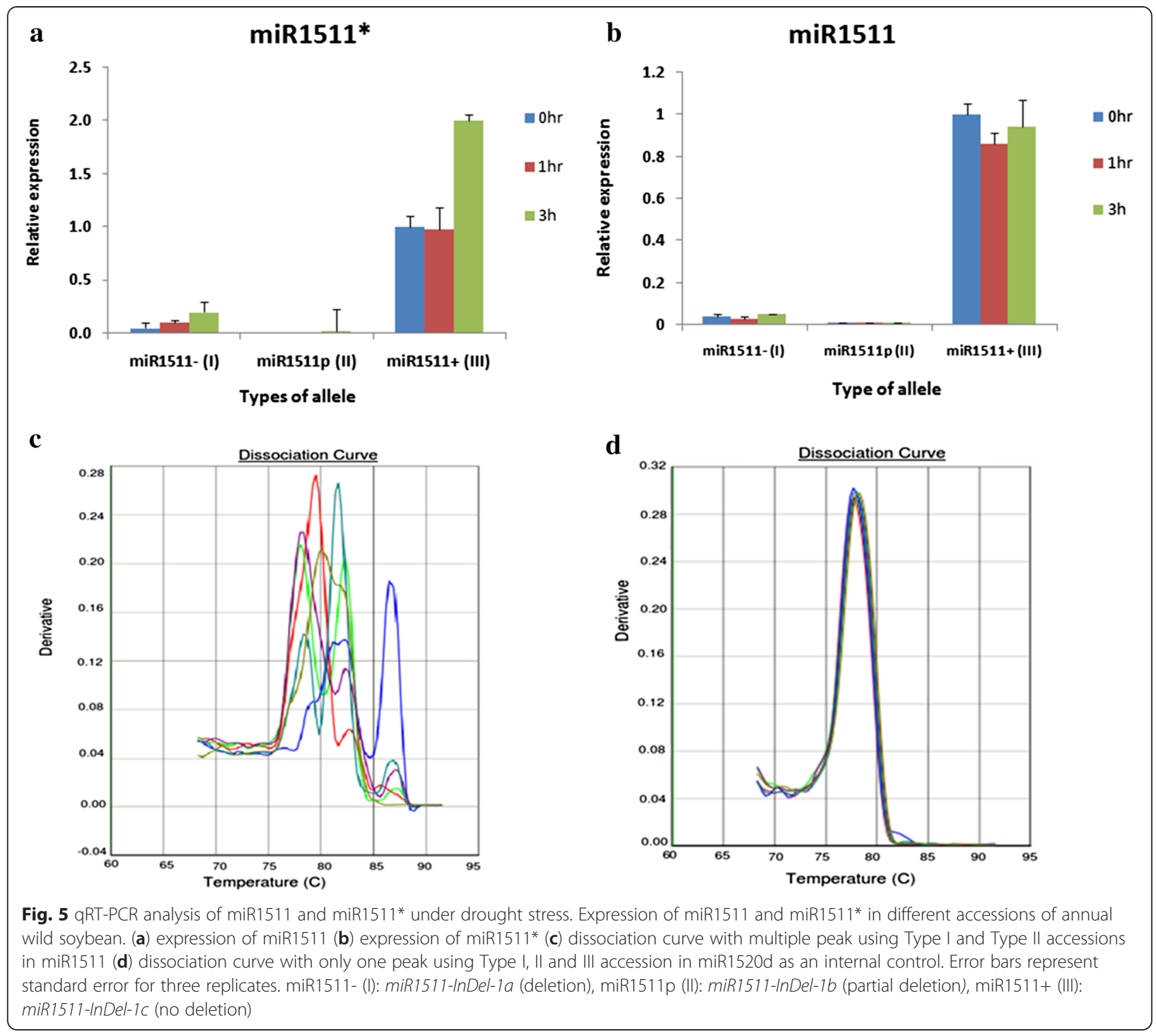

River basin (Huanghuai) had a complete deletion of mature miR1511. According to phylogenetic tree (Fig. 2), we hypothesize that miR1511 is missing in the most ancestral form of G. soja and was gained in later G. sojas and then passed through the domestication bottleneck to the domesticated G. max. It has been suggested that domestication of cultivated soybean was directional selection as soybean is a short- day plant that is adapted to a narrow geographic range, accessions adapted to certain geography would have difficulties in adapting to various eco-regions in breeding especially for yieldrelated traits [55]. In addition, there were two whole genome duplication events in soybean [2], and with subsequent gene loss. This study attempted to infer the genetic variation and evolutionary relationship within the genus Glycine using a functional miRNA marker.

\section{Origin of soybean genetic diversity}

Soybean originated from China where the cultivation of soybean has a long history of more than 5000 years, notably the agricultural ancestor Houji planted five crops including soybean [56]. Many scholars have pointed out different geographic origins of soybean in China including the Northeast region, the Yellow River valley region (Huanghuai) and the South region [57-59]. In this study, the phylogenetic tree analysis of allelic variants from miR1511 allelic pointed out that partial or non missing forms of miR1511 in miR1511InDel-1b and miR1511-InDel-1c are likely to have originated from the missing form of miR1511-InDel-1a which was mainly distributed in the Yellow River basin (Huanghuai) (Fig. 3). Therefore, we suggest that the Yellow River basin (Huanghuai) is the primary centre 
of species diversity as supported by miR1511. Similarly, the highest diversity has been observed for ten quality traits and five quantitative traits and SSR analyze in seven clusters of 23,587 soybean landraces [59] in this region. A SSR and SNP analyses in 303 accessions of domesticated soybean and its wild progenitor indicated that G.max was originated from regions along the Yellow River of China [16]. Thus, the distribution of allelic diversity of miR1511-InDel provides more molecular evidence for the domestication of soybean in the yellow river basins (Huanghuai).

\section{Conclusions}

In conclusion, the functional miR1511 marker (miR1511InDel) was localized within a functional region and found to be useful to analyze genetic variation within Glycine. This was done using simple equipment and standard conditions with low cost. The marker can also be used as a tool for further analysis of phylogenetic relationships using the flanking region of miR1511 in this crop and those of model legumes. Our study suggested that the InDel marker of miR1511 can help to provide a reference for studying genetic diversity, genotyping of germplasm, evolution and the center of species diversities in domestication of soybean and that this marker system could be useful for many applications in breeding and ecology/evolution.

\section{Methods}

\section{Plant materials}

We collected a total of 1,669 accessions representing cultivated soybean [G. $\max ($ L.) Merr.] $(1,206)$ (Additional file 6: Table S1) and wild progenitor species [G. soja Sieb. et Zucc] (463) (Additional file 7: Table S2). The cultivated soybean including the minicore collection [60] were collected from Northeast region, North region, Huanghuai region (Yellow River basin) and South region and 10 outgroup accessions collected outside of China. The wild progenitor species originated from three geographical regions of China, Japan, Korea and Russia. Four perennial wild soybean Glycine microplylla, Glycine tabacine, Glycine latifolia and Glycine tomentella were used for post-PCR sequence alignment. All the accessions within China were selected to represent the geographical range from 23.7 to $51.4^{\circ} \mathrm{N}$ and 106.4 to $131.5^{\circ} \mathrm{E}$.

\section{Bioinformatics data and data analysis}

Soybean genome sequences were obtained from the Phytozome database (http://www.phytozome.net/). The Primer Premier Version 5.00 software package (Premier Biosoft International) was used to design primers and Multalin (http://multalin.toulouse.inra.fr/multalin/ multalin.html) for sequence alignments. An unrooted phylogenetic tree was constructed using MEGA 4.0 with the maximum likelihood (ML) method and bootstrap tests carried out with 1000 iterations [61]. The predicted sequence of pre-miR1511from other crops were confirmed for present of mature sequences and their secondary structure predicted using the RNAfold program (http://mfold.rna.albany.edu/?q= mfold). The Shannon diversity index was calculated as $\mathrm{H}=-$ sum (Pi $\ln [\mathrm{Pi}]$ ) where $\mathrm{H}$ represents the Shannon diversity index and $\mathrm{Pi}$ is the number of individuals in a particular species type divided by the total number of individuals of all types of species in the community.

\section{Genomic DNA extraction and sequencing}

Genomic DNA was extracted using Genomic DNA Purification Kits (Thermo Scientific (Cat \# K0512) according to manufacturer's protocol. All the extracted DNA samples were normalized to $20 \mathrm{ng} / \mu \mathrm{l}$ for PCR amplification. MIR1511 gene fragment amplification primer sequences were designed as follows: MIR1511F, 5'-TCTTCATG GACTTATTTGCCACTTC- 3'; MIR1511R, 5'- CCTTCA AGACCAAATGCTAAA T C G- 3'. The PCR reactions performed with Ex Taq kit (Takara,Dalian,China) with the following cycling parameters: initial denaturing at $94{ }^{\circ} \mathrm{C}$ for $4 \mathrm{~min}, 35$ cycles of denaturing at $94{ }^{\circ} \mathrm{C}$ for $30 \mathrm{~s}$, annealing at $56^{\circ} \mathrm{C}$ for $30 \mathrm{~s}$, extension at $72{ }^{\circ} \mathrm{C}$ for $30 \mathrm{~s}$ and final extension at $72{ }^{\circ} \mathrm{C}$ for $8 \mathrm{~min}$. The PCR product was detected by electrophoresis on $1.5 \%$ agarose gels and the expected fragments were recovered by using a DNA gel extraction kit (Axygen Biotechnology, Hangzhou, China). The purified fragments were ligated into the pMD18-T vector (TaKaRa, Dalian, China) at $16^{\circ} \mathrm{C}$ for $1 \mathrm{~h}$ followed by transformation into Escherichia coli Top10 competent cells (Tiangen Biotech, Beijing, China) and spread onto LB agar plates containing $100 \mu \mathrm{g} / \mathrm{mL}$ ampicillin. The plates were incubated at $37{ }^{\circ} \mathrm{C}$ for $12-16 \mathrm{~h}$. Randomly selected colonies were cultured in liquid LB medium containing $100 \mu \mathrm{g} / \mathrm{mL}$ ampicillin at $37^{\circ} \mathrm{C}$ on an oscillator for $6 \mathrm{~h}$. Positive recombinant clones were screened by colony PCR. PCR products containing the expected inserts were sequenced using M13 forward and reverse primers (GENEWIZ Beijing, China).

\section{RNA isolation and Northern blot analysis}

Total RNA was extracted using an RNAiso plus kit (Takara, Dalian, China) according to manufacturer's protocol and small RNA enrichment was performed as previously described [62]. $40 \mu \mathrm{g}$ of enriched small RNA was separated in a $15 \%$ polyacrylamide gel with $8 \mathrm{M}$ urea, in MOPS buffer ( $\mathrm{pH} 7.0$ ), along with the microRNA marker (New England Biolabs, Ipswich, UK). The samples were electroblotted to positively charged nylon membrane (Amersham Life Science, 
Buckinghamshire, UK) using a semi dry transfer cell (BioRad Laboratories, Richmond, CA, USA). The nylon membrane was again placed onto the Stratagene UV Cross linker (UPV, San Gabriel, CA, USA) at $1200 \mathrm{~kJ}$ (front, back and front for $1 \mathrm{~min}$ each), baked at $80{ }^{\circ} \mathrm{C}$ for $30-60 \mathrm{~min}$ and then prehybridized at $42{ }^{\circ} \mathrm{C}$ in ULTRAhyb- Oligo buffer (Ambicon the membrane at, Austin, TX, USA) for $2-4 \mathrm{~h}$ in a standard rotating hybridization oven. To detect miRNA1511, short oligonucleotides probe (Nor_1511), 5'-CCATGGTATCAG AGCCT GGTT- 3'; the U6 snRNA probe (Nor_U6), 5'- GACCATTTCTCGATTTGTGCG TGTC- 3' were end-labeled with $\gamma \mathrm{P}^{32}$-ATP (PerkinElmer, Waltham, MA, USA) overnight at $37{ }^{\circ} \mathrm{C}$, using T4 polynucleotide kinase (USB Corp, Cleveland, OH, USA). After hybridization, the membrane was washed at $42{ }^{\circ} \mathrm{C}$ using a $2 x S S C+0.5 \%$ SDS solution until radioactivity was reduced to less than $300 \mathrm{cpm}$. The hybridized membrane was exposed to a storage phosphorimager screen (GE Healthcare, Milwaukee, WI, USA) and scanned using FX Pro Plus (BioRad Laboratories, Hercules, CA, USA).

\section{Quantitative real-time PCR}

The following accessions were used for miR1511 and miR1511"gene expression analysis. Type I (miR1511InDel-1a) ZYD03386, which loss mature sequence of miR1511, type II (miR1511-InDel-1b) ZYD04366 which loss partial sequence of miR1511 and miR1511* and type III (miR1511-InDel-1c) ZYD03960 which have miR1511 and miR1511* were used. Total RNA were extracted from the leaves of $\mathrm{V} 2$ stage of seedlings treated by drought (paper dry) at $0,1,3 \mathrm{~h}$ respectively using RNAiso Plus (Takara, Dalian, China) reagent followed by the manufacturer's instructions.

First-strand cDNA synthesis of miRNA was then performed using a miRcute miRNA first-strand cDNA synthesis kit (Tiangen, Beijing, China) according to the manufacturer's instructions. Relative quantification of miRNA expression was carried out using PRISN 7300 real- time PCR system (Applied Biosystems, Foster City,CA, USA) with SYBR Green miRcute miRNA qPCR Detection Kit (Tiangen, Beijing, China) which contained antisense adaptor primers and applying the corresponding miRNA sequences as sense primers. Soybean miR1520d was used as an internal standard. The data were analyzed using the $2^{-\Delta \Delta C t}$ method. The PCR primers for $\mathrm{qPCR}$ of miR1511, miR1511* and miR1520d were as follows: miR1511 primer (5' - GGAACCAGGCTCT GATACCATGG -3'), miR1511* (5' - CCGTGGTAT CAGG TCCTGC TTCA-3') and miR1520d primer (5' ATCAGAACATGACACGTGACAA-3'). The experiment was repeated three times.

\section{Availability of Supporting Data section}

All the supporting data are included as additional files.

\section{Additional files}

Additional file 1: Figure S1. Predict sequence of stem loop miR1511. Predict sequence of stem loop miR1511 in various organisms, underline represents mature sequence.

Additional file 2: Figure S2. Stem loop miR1511 structure by RNA folding in various organism. Pre miRNA stem loop structure by RNA folding (http://mfold.rna.albany.edu/?q=mfold) (a) L. japonica (b) G. max (c) P. tricocarpa (d) M. truncatula (e) V. vinifera.

Additional file 3: Figure S3. Alignment of precursor of miR1511 with pan-genome accessions. Alignment of precursor sequence of miR1511 in cultivated soybean G. max, seven G. soja accessions in pan- genome and P. vulgaris.

Additional file 4: Figure S4. Anti expression of miR1511/miR1511* and its target gene GmRPL4a under stresses condition. QRT-PCR was performed using Type III, miR1511-InDel-1c accessions (a) expression of miR1511* and miR1511 under drought stress (b) expression of GMRPL4a under drought stress [target cleavage site of both miR1511* (GMRPL4*) and miR1511 (GMRPL4)] (c) expression of miR1511* and miR1511 under salt stress (d) expression of GMRPL4a under salt stress [target cleavage site of both miR1511* (GMRPL4*) and miR1511 (GMRPL4)]. Error bars represent standard error for three replicates.

Additional file 5: Figure S5. Alignment of miR1511 target gene with pan-genome accessions. Alignment of miR1511 target gene among G.max, seven G.soja from pan genome and P. vulgaris (a) tail region of the target gene (b) miR1511* complementary in target gene region (c) miR1511 complementary in target gene region.

Additional file 6: Table S1. List of cultivated soybean. List of the cultivated soybean (G. max) accessions used in this study.

Additional file 7: Table S2. List of wild soybean. List of the annual wild soybean (G. soja) accessions used in this study. I: miR1511-InDel-1a; II: miR1511-InDel-1b; III: miR1511-InDel-1C.

\section{Competing interests}

The authors declare that they have no competing interests.

\section{Authors' contributions}

NMPSH participated in the conception of the study, carried out the genotyping, performed statistic, bioinformatics, qRT-PCR analysis and drafted the paper. ZQL participated in marker development. LGJ verified existence of miR1511 by northern blot together with ZQL. BN analyzed consistency of absence of miR1511 in G. soja D (Huanghuai) in pan-genome. KJW supported some of the annual wild soybean accession samples used in this study. LJQ conceived of and coordinated the study, interpreted results and co-wrote the paper. All authors read and approved the final manuscript.

\section{Acknowledgements}

This work was carried out with the support of National Natural Science Foundation of China (30871621 and 30490251) and Organization for Women in Science for the Developing World (fund reservation No. 3240237390). We thank Dr. Scott A. Jackson (Institute of Plant Breeding, Genetics \& Genomics, and University of Georgia) for constructive comments and useful suggestions.

\section{Author details}

${ }^{1}$ The National Key Facility for Crop Gene Resources and Genetic Improvement (NFCRI), Institute of Crop Science, Chinese Academy of Agricultural Sciences, 100081 Beijing, China. ${ }^{2}$ Institute of Plant Breeding, Genetics, and Genomics, University of Georgia, Athens, GA, USA.

Received: 27 January 2015 Accepted: 29 May 2015

Published online: 18 June 2015 


\section{References}

1. Hartman GL, West ED, Herman TK. Crops that feed the World 2. Soybean - worldwide production, use, and constraints caused by pathogens and pests. Food Security. 2011;3(1):5-17.

2. Schmutz J, Cannon SB, Schlueter J, Ma J, Mitros T, Nelson W, et al. Genome sequence of the palaeopolyploid soybean. Nature. 2010;463(7278):178-83.

3. Rajcan I, Hou G, Weir AD. Advances in Breeding of Seed-Quality Traits in Soybean. J Crop Improv. 2005;14(1-2):145-74.

4. Kalendar R, Flavell AJ, Ellis TH, Sjakste T, Moisy C, Schulman AH. Analysis of plant diversity with retrotransposon-based molecular markers. Heredity. 2011;106(4):520-30.

5. Poczai P, Varga I, Laos M, Cseh A, Bell N, Valkonen JP, et al. Advances in plant gene-targeted and functional markers: a review. Plant Methods. 2013;9(1):6.

6. Botstein D, White RL, Skolnick M, Davis RW. Construction of a genetic linkage map in man using restriction fragment length polymorphisms. Am J Hum Genet. 1980;32(3):314-31.

7. Williams JG, Kubelik AR, Livak KJ, Rafalski JA, Tingey SV. DNA polymorphisms amplified by arbitrary primers are useful as genetic markers. Nucleic Acids Res. 1990;18(22):6531-5.

8. Vos P, Hogers R, Bleeker M, Reijans M, van de Lee T, Hornes M, et al. AFLP. a new technique for DNA fingerprinting. Nucleic Acids Res. 1995;23(21):4407-14.

9. Wang Z, Weber JL, Zhong G, Tanksley SD. Survey of plant short tandem DNA repeats. Theor Appl Genet. 1994;88(1):1-6.

10. Syvanen AC. Accessing genetic variation: genotyping single nucleotide polymorphisms. Nat Rev Genet. 2001;2(12):930-42.

11. Gupta PK, Rustgi S. Molecular markers from the transcribed/expressed region of the genome in higher plants. Funct Integr Genomics. 2004;4(3):139-62

12. Cregan $P$, Jarvik T, Bush A, Shoemaker R, Lark K, Kahler A, et al. An integrated genetic linkage map of the soybean genome. Crop Sci. 1999:39(5):1464-90

13. Song QJ, Marek LF, Shoemaker RC, Lark KG, Concibido VC, Delannay X, et al. A new integrated genetic linkage map of the soybean. Theor Appl Genet. 2004;109(1):122-8

14. Hyten DL, Choi I-Y, Song Q, Specht JE, Carter TE, Shoemaker RC, et al. A High Density Integrated Genetic Linkage Map of Soybean and the Development of a 1536 Universal Soy Linkage Panel for Quantitative Trait Locus Mapping. Crop Sci. 2010;50(3):960.

15. Xie H. Genetic diversity of Chinese summer soybean germplasm revealed by SSR markers. Chin Sci Bull. 2005;50(6):526.

16. Li YH, Li W, Zhang C, Yang L, Chang RZ, Gaut BS, et al. Genetic diversity in domesticated soybean (Glycine max) and its wild progenitor (Glycine soja) for simple sequence repeat and single-nucleotide polymorphism loci. New Phytol. 2010;188(1):242-53.

17. Cregan P, Mudge J, Fickus E, Marek L, Danesh D, Denny R, et al. Targeted isolation of simple sequence repeat markers through the use of bacterial artificial chromosomes. Theor Appl Genet. 1999;98(6-7):919-28.

18. Li YH, Zhou G, Ma J, Jiang W, Jin LG, Zhang Z, et al. De novo assembly of soybean wild relatives for pan-genome analysis of diversity and agronomic traits. Nat Biotechnol. 2014;32(10):1045-52.

19. Shedlock AM, Okada N. SINE insertions: powerful tools for molecular systematics. Bioessays. 2000;22(2):148-60.

20. Ambros $V$. The functions of animal microRNAs. Nature. 2004:431(7006):350-5.

21. Palatnik JF, Allen E, Wu X, Schommer C, Schwab R, Carrington JC, et al. Control of leaf morphogenesis by microRNAs. Nature. 2003:425(6955):257-63

22. Glazinska P, Zienkiewicz A, Wojciechowski W, Kopcewicz J. The putative miR172 target gene InAPETALA2-like is involved in the photoperiodic flower induction of Ipomoea nil. J Plant Physiol. 2009;166(16):1801-13.

23. Sunkar R, Chinnusamy V, Zhu J, Zhu JK. Small RNAs as big players in plant abiotic stress responses and nutrient deprivation. Trends Plant Sci. 2007;12(7):301-9.

24. Liu HH, Tian X, Li YJ, Wu CA, Zheng CC. Microarray-based analysis of stressregulated microRNAs in Arabidopsis thaliana. RNA. 2008;14(5):836-43.

25. Jian X, Zhang L, Li G, Zhang L, Wang X, Cao X, et al. Identification of novel stress-regulated microRNAs from Oryza sativa L. Genomics. 2010;95(1):47-55.

26. Carrington $\mathrm{JC}$, Ambros $\mathrm{V}$. Role of microRNAs in plant and animal development. Science (New York, NY). 2003;301(5631):336-8.
27. Meyers BC, Axtell MJ, Bartel B, Bartel DP, Baulcombe D, Bowman JL, et al. Criteria for annotation of plant MicroRNAs. Plant Cell. 2008;20(12):3186-90.

28. Kozomara A. Griffiths-Jones S: miRBase: integrating microRNA annotation and deep-sequencing data. Nucleic Acids Res. 2011;39(Database issue):D152-157

29. Gui $Y$, Yan G, Bo S, Tong Z, Wang Y, Xiao B, et al. iSNAP: a small RNA-based molecular marker technique. Plant Breed. 2011;130(5):515-20.

30. Mondal TK, Ganie SA. Identification and characterization of salt responsive miRNA-SSR markers in rice (Oryza sativa). Gene. 2014;535(2):204-9.

31. Subramanian S, Fu Y, Sunkar R, Barbazuk WB, Zhu JK, Yu O. Novel and nodulation-regulated microRNAs in soybean roots. BMC Genomics. 2008;9:160.

32. Arenas-Huertero C, Perez B, Rabanal F, Blanco-Melo D, De la Rosa C, EstradaNavarrete $\mathrm{G}$, et al. Conserved and novel miRNAs in the legume Phaseolus vulgaris in response to stress. Plant Mol Biol. 2009;70(4):385-401.

33. Lelandais-Briere C, Naya L, Sallet E, Calenge F, Frugier F, Hartmann C, et al. Genome-wide Medicago truncatula small RNA analysis revealed novel microRNAs and isoforms differentially regulated in roots and nodules. Plant Cell. 2009:21(9):2780-96.

34. Pantaleo V, Szittya G, Moxon S, Miozzi L, Moulton V, Dalmay T, et al. Identification of grapevine microRNAs and their targets using highthroughput sequencing and degradome analysis. Plant J. 2010;62(6):960-76.

35. Luo Z, Jin L, Qiu L. MiR1511 co-regulates with miR1511* to cleave the GmRPL4a gene in soybean. Chin Sci Bull. 2012;57(28-29):3804-10.

36. Unver T, Namuth-Covert DM, Budak H. Review of current methodological approaches for characterizing microRNAs in plants. Int J Plant Genom. 2009;2009:262463.

37. Rosado A, Li R, van de Ven W, Hsu E, Raikhel NV. Arabidopsis ribosomal proteins control developmental programs through translational regulation of auxin response factors. Proc Natl Acad Sci. 2012:109(48):19537-44.

38. Trifa Y, Privat I, Gagnon J, Baeza L, Lerbs-Mache S. The nuclear RPL4 gene encodes a chloroplast protein that co-purifies with the T7-like transcription complex as well as plastid ribosomes. J Biol Chem. 1998;273(7):3980-5.

39. Lee RC, Feinbaum RL, Ambros V. The $C$. elegans heterochronic gene lin- 4 encodes small RNAs with antisense complementarity to lin-14. Cell. 1993:75(5):843-54

40. Pasquinelli $A E, M c C o y ~ A$, Jimenez E, Salo E, Ruvkun G, Martindale $M Q$, et al Expression of the 22 nucleotide let-7 heterochronic RNA throughout the Metazoa: a role in life history evolution? Evol Dev. 2003:5(4):372-8.

41. Pasquinelli AE, Reinhart BJ, Slack F, Martindale MQ, Kuroda MI, Maller B, Hayward DC, Ball EE, Degnan B, Muller P et al. Conservation of the sequence and temporal expression of let-7 heterochronic regulatory RNA. In: Nature. vol. 408, 2000/11/18 edn; 2000: 86-89.

42. Tanzer A, Stadler PF. Molecular evolution of a microRNA cluster. J Mol Biol. 2004;339(2):327-35.

43. Ding D, Zhang L, Wang H, Liu Z, Zhang Z, Zheng Y. Differential expression of miRNAs in response to salt stress in maize roots. Ann Bot. 2009:103(1):29-38.

44. Lagos-Quintana M, Rauhut R, Yalcin A, Meyer J, Lendeckel W, Tuschl T. Identification of Tissue-Specific MicroRNAs from Mouse. Curr Biol. 2002;12(9):735-9.

45. Kim J, Krichevsky A, Grad Y, Hayes GD, Kosik KS, Church GM, et al. Identification of many microRNAs that copurify with polyribosomes in mammalian neurons. Proc Natl Acad Sci U S A 2004;101(1):360-5

46. Zhu QH, Spriggs A, Matthew L, Fan L, Kennedy G, Gubler F, et al. A diverse set of microRNAs and microRNA-like small RNAs in developing rice grains. Genome Res. 2008;18(9):1456-65.

47. Szittya G, Moxon S, Santos DM, Jing R, Fevereiro MP, Moulton V, et al. Highthroughput sequencing of Medicago truncatula short RNAs identifies eight new miRNA families. BMC Genomics. 2008:9:593.

48. Cuperus JT, Fahlgren N, Carrington JC. Evolution and functional diversification of MIRNA genes. Plant Cell. 2011;23(2):431-42.

49. Floyd SK, Bowman JL. Gene regulation: Ancient microRNA target sequences in plants. Nature. 2004:428(6982):485-6.

50. Allen E, Xie Z, Gustafson AM, Sung GH, Spatafora JW, Carrington JC. Evolution of microRNA genes by inverted duplication of target gene sequences in Arabidopsis thaliana. Nat Genet. 2004;36(12):1282-90.

51. Piriyapongsa J, Jordan IK. Dual coding of siRNAs and miRNAs by plant transposable elements. RNA. 2008;14(5):814-21. 
52. Felippes FF, Schneeberger K, Dezulian T, Huson DH, Weigel D. Evolution of Arabidopsis thaliana microRNAs from random sequences. RNA. 2008;14(12):2455-9.

53. Xu D, Abe J, Gai J, Shimamoto Y. Diversity of chloroplast DNA SSRs in wild and cultivated soybeans: evidence for multiple origins of cultivated soybean. Theor Appl Genet. 2002;105(5):645-53.

54. Lam HM, Xu X, Liu X, Chen W, Yang G, Wong FL, et al. Resequencing of 31 wild and cultivated soybean genomes identifies patterns of genetic diversity and selection. Nat Genet. 2010;42(12):1053-9.

55. Qiu LJ, Xing LL, Guo Y, Wang J, Jackson SA, Chang RZ. A platform for soybean molecular breeding: the utilization of core collections for food security. Plant Mol Biol. 2013;83(1-2):41-50.

56. Qiu LJ, Chen PY, Liu ZX, Li YH, Guan RX, Wang LH, et al. The worldwide utilization of the Chinese soybean germplasm collection. Plant Genet Resour-C. 2011;9(1):109-22

57. Dong YS, Zhao LM, Liu B, Wang ZW, Jin ZQ, Sun H. The genetic diversity of cultivated soybean grown in China. Theor Appl Genet. 2004;108(5):931-6.

58. Wen Z, Ding Y, Zhao T, Gai J. Genetic diversity and peculiarity of annual wild soybean (G. soja Sieb. et Zucc.) from various eco-regions in China. Theor Appl Genet. 2009;119(2):371-81.

59. Li Y, Guan R, Liu Z, Ma Y, Wang L, Li L, et al. Genetic structure and diversity of cultivated soybean (Glycine max (L.) Merr.) landraces in China. Theor Appl Genet. 2008;117(6):857-71.

60. Qiu LJ, Li YH, Guan RX, Liu ZX, Wang LX, Chang RZ. Establishment, Representative Testing and Research Progress of Soybean Core Collection and Mini Core Collection. Acta Agron Sin. 2009;35(4):571-9.

61. Tamura K, Dudley J, Nei M, Kumar S. MEGA4: Molecular Evolutionary Genetics Analysis (MEGA) software version 4.0. Mol Biol Evol. 2007;24(8):1596-9.

62. Sunkar R, Zhu JK. Novel and stress-regulated microRNAs and other small RNAs from Arabidopsis. The Plant cell. 2004;16(8):2001-19.

\section{Submit your next manuscript to BioMed Central and take full advantage of:}

- Convenient online submission

- Thorough peer review

- No space constraints or color figure charges

- Immediate publication on acceptance

- Inclusion in PubMed, CAS, Scopus and Google Scholar

- Research which is freely available for redistribution 